\title{
Vortex fluidic mediated one-step fabrication of polyvinyl alcohol hydrogel films with tunable surface morphologies and enhanced self-healing properties
}

\author{
Javad Tavakoli ${ }^{1,2^{*}}$, Colin L. Raston ${ }^{2}$, Yong $\mathrm{Ma}^{3,2}$ and Youhong Tang ${ }^{2^{*}}$
}

\begin{abstract}
Previous strategies for controlling the surface morphologies of polyvinyl alcohol (PVA)-based hydrogels, including freeze-drying and electrospinning, require a posttreatment process, which can affect the final textures and properties of the hydrogels. Of particular interest, it is almost impossible to control the surface morphology during the formation of PVA hydrogels using these approaches. The strategy reported in this study used the novel vortex fluidic device (VFD) technology, which for the first time provided an opportunity for one-step fabrication of PVA hydrogel films. PVA hydrogels with different surface morphologies could be readily fabricated using a VFD. By also reducing the crosslinking agent concentration, a self-healing gel with enhanced fracture stress $(60 \%$ greater than that of traditionally made hydrogel) was achieved. Interestingly, the associated selfhealing property remained unchanged during the 260 -s mechanical testing performed with the strain rate of $5 \% \mathrm{~s}^{-1}$. The VFD can effectively tune the surface morphologies of the PVA-based hydrogels and their associated properties, particularly the self-healing property.
\end{abstract}

Keywords: polyvinyl alcohol, vortex fluidic device, one-step fabrication, self-healing, surface morphology manipulation

\section{INTRODUCTION}

Polyvinyl alcohol (PVA) is a commercially available hydrophilic linear polymer produced via hydrolysis of poly(vinyl acetate). Therefore, its physicochemical properties, including viscosity, adhesion, mechanical strength and formability, depend primarily on the molecular weight of poly(vinyl acetate) and the associated degree of hydrolysis [1]. Being chemically inert and stable, PVA is considered safe and its ability to take up water augments its associated biocompatibility. The degree of hydrolysis determines the swelling capacity of the hydrogel, classifying PVA into a low, medium, or highly swellable biomaterial [2]. Directly relevant to the degree of hydrolysis, the number of $\mathrm{OH}$ groups in the PVA molecular structure generally offers crystallization accounting for the physical crosslinking of the hydrogel networks [3]. A highly crystallized PVA hydrogel has low swelling property, making it desirable for specific biomedical and pharmaceutical applications [4-6]. Different studies have shown the notable performance of PVA hydrogel and its composites for wound healing [7], sensors and electrodes $[8,9]$, tissue engineering [10-12] and biomedical applications [13-15].

Although physically crosslinked PVA hydrogels are highly biocompatible, a major drawback is the lack of an appropriate strategy to properly tune the associated mechanical and physicochemical properties. Application of freeze-thaw cycles has been the sole approach used to enhance the mechanical properties, which diversely affects the swelling capacity [16-18]. On the other hand, the final properties of a PVA hydrogel, including structural stability, mechanical strength, swelling capacity and rate of drug release, can be controlled by the use of chemical crosslinking agents (i.e., formaldehyde, glutaraldehyde and ketones) [19-21]. However, that method is toxic, and the biocompatibility of the hydrogel is strongly influenced by the amount of residual crosslinker. Clearly, despite the hydrogel achieving desirable properties via chemical crosslinking, the increase in the level of toxicity alongside the complex relevant purification strategies compromises the advantages. Recent studies have revealed that esterification or the formation of hydrogen bonds via the

\footnotetext{
${ }^{1}$ School of Biomedical Engineering, University of Technology Sydney, New South Wales 2007, Australia

${ }^{2}$ Institute for NanoScale Science and Technology, College of Science and Engineering, Flinders University, South Australia 5042, Australia

${ }^{3}$ School of Sports Engineering and Information Technology, Wuhan Sports University, Hubei 430079, China

* Corresponding authors (emails: youhong.tang@flinders.edu.au (Tang Y); javad.tavakoli@uts.edu.au (Tavakoli J))
} 
employment of boric acid including borax can form an insoluble PVA structure $[22,23]$. That method opens new avenues for the fabrication of biocompatible PVA hydrogels with adjustable physicochemical and mechanical properties for different applications [24-27]. Borax has a long history of medical applications, with the mean lethal dose exceeding $700 \mathrm{mg} \mathrm{kg}^{-1}$ in humans with no toxicity reported [28]. Cytotoxicity screening of borax using different cells has shown a non-toxic nature in several studies [23,24,29]. Clearly, among different methods for crosslinking PVA-based hydrogels, the employment of borax is a simple approach without the excessive imposition of controls over the final associated properties [30-32]. The approach effectively supports the fabrication of PVA hydrogel composites with different properties ranging from self-adhesion to high mechanical strength, with negligible side effects on biomedical properties [3335].

To the best of the authors' knowledge, independent of the crosslinking method, construction of a bulk PVA hydrogel containing hierarchical architectures or different surface morphologies is still challenging. The conventional method for fabricating PVA films is solvent casting that not only involves at least two steps but also lacks control over surface morphology. There is a lack in methodology to just control the surface morphology of hydrogel films precisely. Other frequently used methods including electrospinning or freeze-drying, which lead to a random fibrous or porous structure, are mainly recruited to control the bulk morphology [36]. These methods require post-crosslinking modification to achieve structural stability and mechanical performance.

In this study, for the first time, we develop a simple one-step method to fabricate PVA hydrogels with different surface morphologies that are not attainable using other fabrication approaches. To investigate this novel concept, a recently developed vortex fluidic device (VFD) was employed (Fig. 1a). The VFD is a relatively inexpensive research tool for controlling chemical reactivity and selectivity, materials synthesis, and probing the structure of self-organized systems, offering a range of benefits over conventional processing [37,38]. Dynamic thin film in the VFD platform is generated in a rapidly rotating surface, imparting high shear stress and micromixing. Typically, a quartz or glass tube closed at one end is rapidly rotated at a $45^{\circ}$ tilt angle, with a finite amount of liquid in the tube in the confined mode of the VFD or, where liquids are constantly fed into the tube, exiting at the top, as the continuous flow mode of operation. We

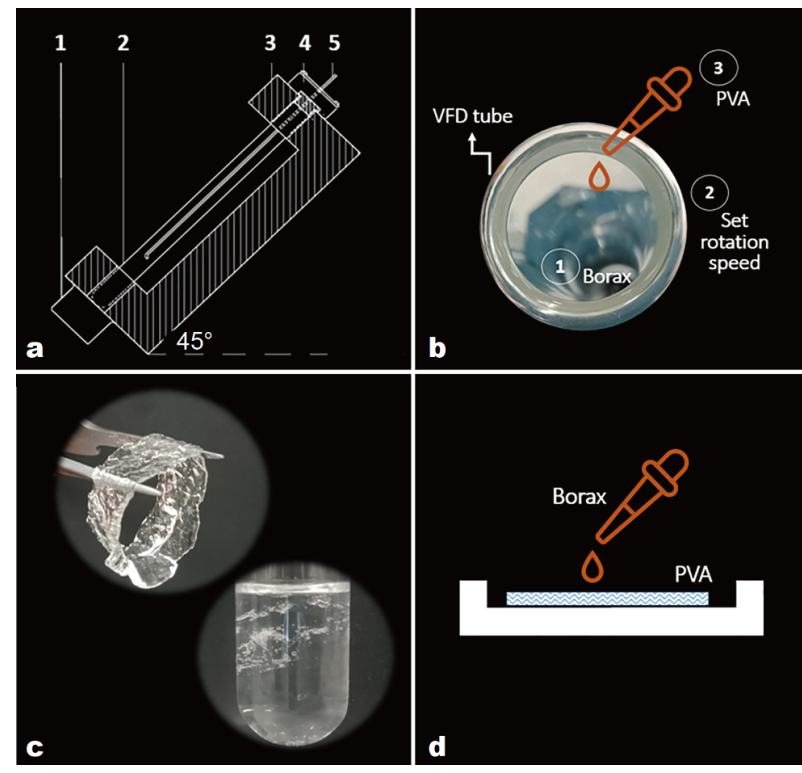

Figure 1 (a) Schematic of a VFD, highlighting 1, an electric motor; 2, a VFD tube ( $20 \mathrm{~mm}$ diameter); 3 , a tube holder; 4 , a tube cap; and 5 , a jet feed inlet, (b) our new approach for the one-step fabrication of PVA hydrogel using VFD to control the surface morphology, (c) photographs of a PVA hydrogel (top) and self-healing PVA gel (bottom) formulated with different concentrations of borax solution, and (d) the conventional approach for fabrication of PVA films.

have successfully employed VFD for a number of diverse applications, including the fabrication of various nanocarbon materials [39,40], intensified aqueous two-phase separation for protein purification [41], manipulation of polymer networks [42], exfoliation of graphite and boron nitride [43] and protein folding [44]. Of particular interest, we have found the VFD to be effective for controlling the size and shape of nanoparticles in both topdown and bottom-up processing [45]. In contrast to other methods incorporating rotation to evaporate a solvent and form a film, such as spin coating method, the VFD method imparts micro-mixing and shear stress and provides a variety of controlling parameters, including type of solvents, rotation, and direction of speed, temperature, rate of injection, tilt angle, and modes of operation, i.e., confined and continuous flow to simultaneously fabricate a hydrogel film and tune the surface morphology.

\section{EXPERIMENTAL SECTION}

\section{Materials and reagents}

PVA (molecular weight $=110,000$; hydrolysed $>99 \%$ ) and sodium tetraborate (borax; assay 99\%) were purchased from Sigma-Aldrich and used without further purification. 


\section{Sample preparation}

PVA $(6 \% w / w)$ solution was prepared by adding the powder to distilled water at $90^{\circ} \mathrm{C}$ with constant stirring (1000 rpm) overnight in a sealed container. Borax solution $\left(100 \mathrm{mmol} \mathrm{L}^{-1}\right)$ was prepared by constant stirring of borax powder in water at $50^{\circ} \mathrm{C}$ for $15 \mathrm{~min}$. Both solutions were kept sealed for sample fabrication.

For the one-step fabrication of PVA hydrogels, the VFD tube was filled with $2 \mathrm{~mL}$ of the borax solution and VFD rotation speeds were set within the 1000-5000 rpm range. Then, using a $5 \mathrm{~mL}$ syringe (gauge 18), the PVA solution was injected into the VFD using an inlet feed jet (Fig. 1b). The formation of hydrogels at different rotation speeds was continued for 5 min before extraction (Fig. 1ctop) and the PVA hydrogels were dried at room temperature for further assessment. Also, a PVA film was fabricated using the conventional solvent casting method (Fig. 1d). Briefly, the PVA solution was cast in a petri dish and placed at room temperature. Upon evaporation of water, the borax solution was added to the petri dish to crosslink the PVA. Also, at the constant rotation speed of $4000 \mathrm{rpm}$, the effects of time lapse and feed jet size were investigated. A high-speed camera (V1212, DANTEC Dynamics) with a $35 \mathrm{~mm}$ lens was used to capture transient phenomena at 12,600 fps within the VFD tube upon injection of the PVA solutions. Further, the concentration of the borax solution was reduced to $50 \mathrm{mmol} \mathrm{L}^{-1}$ to fabricate self-healing PVA gels, using both VFD (Fig. 1cbottom) and conventional methods (mixing using a magnet stirrer) and the results were compared for selfadhesion properties.

\section{Characterizations}

Scanning electron microscopy (SEM) imaging (Inspect F50, FEI Company, USA) was performed to analyze the surface morphologies of the PVA films by placing them on an aluminium stub. The PVA films were sputtercoated with platinum to $2 \mathrm{~nm}$ thickness. To compare the self-adhesion strengthes of the VFD-driven PVA gels with that of hydrogels made by the conventional method, a micromechanical machine (Cellscale, Canada) was employed. Both VFD-driven and conventionally made PVA hydrogels were cast in a petri dish with a thickness of $2 \mathrm{~mm}$. Then a 10 -mm-width gel was separated from the centre of the petri dish after $10 \mathrm{~min}$ and cut in half. Each half was attached to the CellScale actuator, secured with a small lab-made gripper while they were not attached. The actuators were moved slowly to attach the gels at the cutting edge until the compression force reached $30 \mathrm{mN}$. Then, the displacement control tensile test was executed at the strain rate of $5 \% \mathrm{~s}^{-1}$ for $260 \mathrm{~s}$. During the test, images were captured at the frequency of $1 \mathrm{fps}$ using the CellScale camera. This set-up remained unchanged for both VFD-driven and conventionally made PVA hydrogels. Changes in fracture stress were measured as a function of time for $260 \mathrm{~s}$.

\section{RESULTS AND DISCUSSION}

\section{Tuning PVA hydrogel film surface morphology by VFD rotation speeds}

From the SEM images, no specific pattern was found for the PVA hydrogel films prepared by the conventional method, as expected (Fig. 2a, b). In contrast, PVA hydrogel films prepared using the VFD revealed different

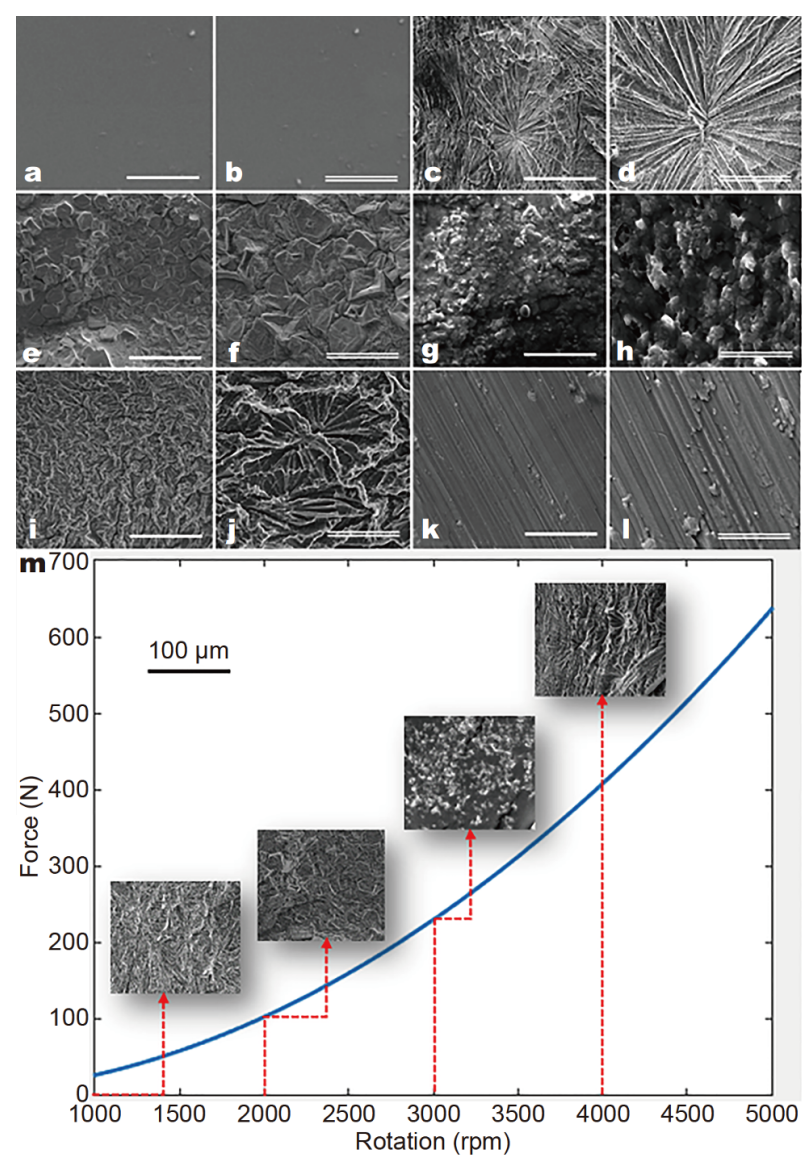

Figure 2 SEM images of the surfaces of PVA hydrogel films fabricated using $(\mathrm{a}, \mathrm{b})$ the traditional method compared with the VFD-driven method at different rotation speeds of (c, d) 1000, (e, f) 2000, (g, h) 3000, $(i, j) 4000$ and $(k, ~ l) 5000 \mathrm{rpm}$. Scale bars represent two magnifications of 200 and $50 \mu \mathrm{m}$. (m) Change in the centrifugal force experienced by PVA film at different rotation speeds which resulted in the formation of different surface morphologies (insets: the images captured from different samples to those presented in $(\mathrm{a}-\mathrm{l})$. 
surface morphologies. At the low rotational speed of $1000 \mathrm{rpm}$, numerous circular patterns with the approximate diameter of 120-150 $\mu \mathrm{m}$ were observed, including radial grooves. Interestingly, the grooves began from the centre, diverging outward to the perimeter of the circles (Fig. 2c, d). At the rotational speed of $2000 \mathrm{rpm}$, PVA films were comprised of flat particles ranging from 10 to $40 \mu \mathrm{m}$ in size with almost irregular shapes. Our detailed observation at higher magnification revealed distinct regions for the particles including a flat or a hollow central part with slightly elevated edges (Fig. 2e, f). When the rotation speed was set to $3000 \mathrm{rpm}$, a cluster of small sphere-like particles $(5-10 \mu \mathrm{m}$ in diameter) were distributed irregularly across the surface, as shown in Fig. $2 \mathrm{~g}$ and $\mathrm{h}$. An increase of the rotation speed to $4000 \mathrm{rpm}$ resulted in the formation of a flower-like pattern across the surface of the hydrogel (Fig. 2i, j), and when the high rotational speed of $5000 \mathrm{rpm}$ was employed, straight parallel grooves $<5 \mu \mathrm{m}$ in width were formed (Fig $2 \mathrm{k}, \mathrm{l}$ ). The forces applied to the PVA solution after injection due to the rotation of the borax solution are likely to be the main cause of these phenomena. The PVA injected into the VFD tube experienced different magnitudes of the centrifugal force, depending on different rotation speeds [45], which was responsible for the formation of different surface morphologies (Fig. $2 \mathrm{~m}$ ). Since these observations identified that the formation of different surface morphologies in PVA hydrogel films depended strongly on the magnitude of the applied centrifugal force generated by the rotation of the VFD tube, it is likely that onestep fabrication of PVA hydrogel films with different surface textures is reproducible. Therefore, the proposed method presents new avenues in the preparation of hydrogel films with customized surface textures. The control of the surface morphology in hydrogels has a potential impact on the development of high-performance devices for biomedical applications, such as tissueengineered scaffolds and systems for drug delivery. Noting that surface morphology provides a unique way of controlling cell-biomaterial interaction and plays a major role in modulating cellular behaviour, such as cell attachment and proliferation during cell culture [46-48].

We found that by precisely varying the rotation speed of the VFD tube, PVA hydrogel films with different surface morphologies were readily fabricated. Similar surface morphologies as a function of rotation speed were seen frequently amongst different batches indicating the repeatability of the process, as shown in Fig. 3. Our observations based on SEM images revealed that the VFD tube plays an important role in the development of a new

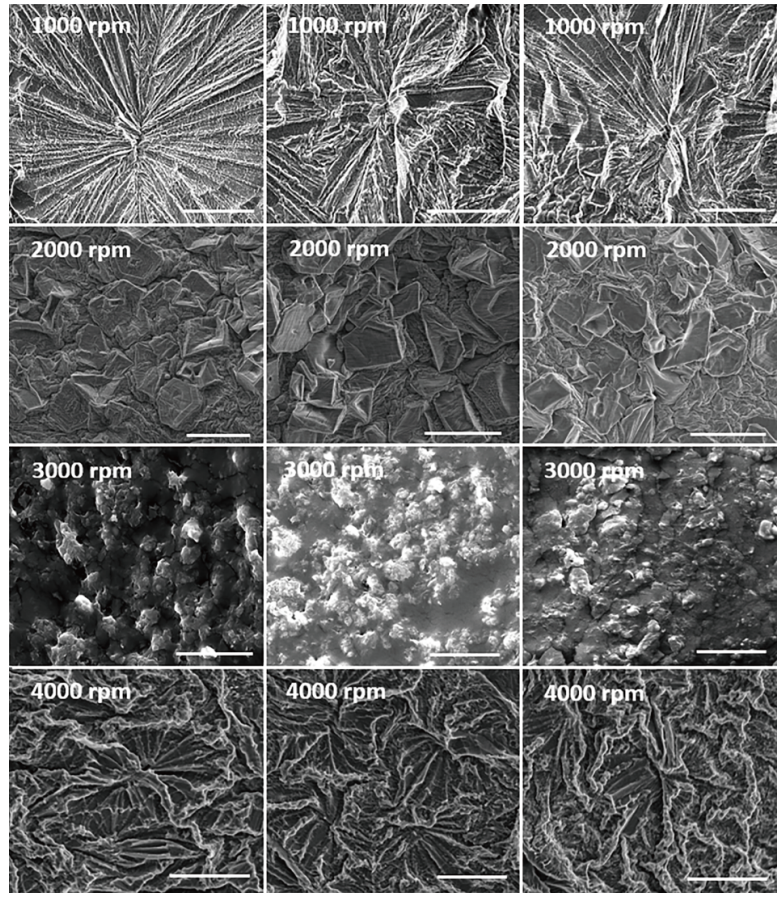

Figure 3 Similar surface morphologies were observed for PVA hydrogels at the same rotation speeds (1000-4000 rpm) for different batches (scale bars: $50 \mu \mathrm{m}$ ).

strategy for one-step fabrication of PVA hydrogel films with different surface architectures. It is likely that this unique approach can be readily extended to the fabrication of other hydrogels that are crosslinked by borax or ionic solutions $[23,49]$.

\section{VFD jet feed inlet effect on PVA hydrogel surface morphology}

In further investigation, the PVA solution was injected into a VFD tube rotating at $4000 \mathrm{rpm}$ using jet feed inlets with two different diameters of $1 \mathrm{~mm}$ and $0.84 \mathrm{~mm}$ (inner diameter of a gauge syringe), as shown in insets in Fig. $4 \mathrm{a}$ and $b$. Interestingly, we found a significant change in the

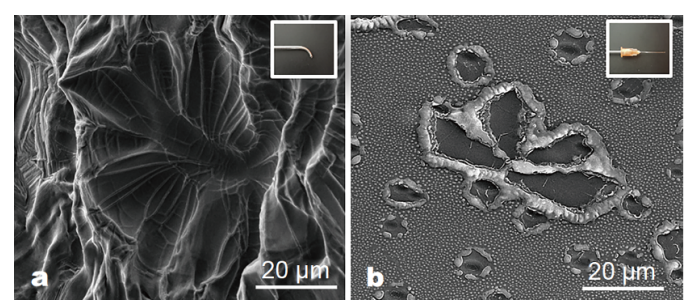

Figure 4 SEM images of the surface of PVA hydrogels fabricated in a rotating VFD tube at $4000 \mathrm{rpm}$ using (a) a $1 \mathrm{~mm}$ diameter and (b) a gauge 18 syringe (inner diameter $0.84 \mathrm{~mm}$ ) jet feeds. The inset images show two different jet feed types. 
surface morphology of the PVA films when the different jet feed inlets were used with two different diameters. The employment of two different jet feeds resulted in the formation of a flower-like structure on the surface of the PVA hydrogel. However, when a needle-like jet feed inlet was used, those structures were smaller with elevated edges and smooth inner regions. A larger flower-like structure containing leaf-like veins was observed when the jet feed with larger diameter was used. When a gauge 18 syringe jet feed was used, the surfaces of the regions outside the 'flowers' seemed to be packed with small spherical structures.

\section{Understanding of the formation mechanism of the VFD-}

\section{driven PVA hydrogel film}

Different strategies were used to obtain deeper insight into the mechanism of PVA hydrogel film formation using VFD. First, images captured during the injection of PVA solution into the VFD tube using a high-speed camera revealed that the PVA droplets were unlikely to turn into or form particles before attaching to the tube surface (Supplementary movie 1). To further investigate this observation, SEM images were captured from both top and back layers of the PVA hydrogel films at different rotation speeds (1000-4000 rpm), as shown in Fig. 5a-c. We found that the back layer of the PVA hydrogel films had a smooth surface without any specific pattern or surface morphology, confirming our observations with the high-speed camera. Then, with the constant rotation speed of $4000 \mathrm{rpm}$, the effect of time lapse on the formation of PVA hydrogel films was explored. In a series of individual experiments, the PVA solution was injected into the borax solution using a $1 \mathrm{~mm}$ jet feed inlet, while the rotation of the VFD tube was set at $4000 \mathrm{rpm}$. At the time points of 1,2 and $4 \mathrm{~min}$, the PVA hydrogel films were extracted and SEM images were captured. As shown in Fig. $5 \mathrm{~d}-\mathrm{f}$, the surface of the hydrogel was subjected to change at different time points. Change in the surface morphology of the PVA hydrogel films initiated at the surface, which is the first region to be exposed to the borax solution. It is believed that, following the injection of PVA solution into the borax solution in the rotating VFD tube, diffusion of the borax into the flattened PVA solution was the main reason for the formation of the PVA hydrogel film. Because the outer region of the flattened PVA solution was the first region to be exposed to the crosslinking agent, it is likely that a core-shell PVA hydrogel film formed with the PVA solution within a soft crosslinked shell. Later, the progress of the diffusion process resulted in crosslinking of the bulk PVA hydro-
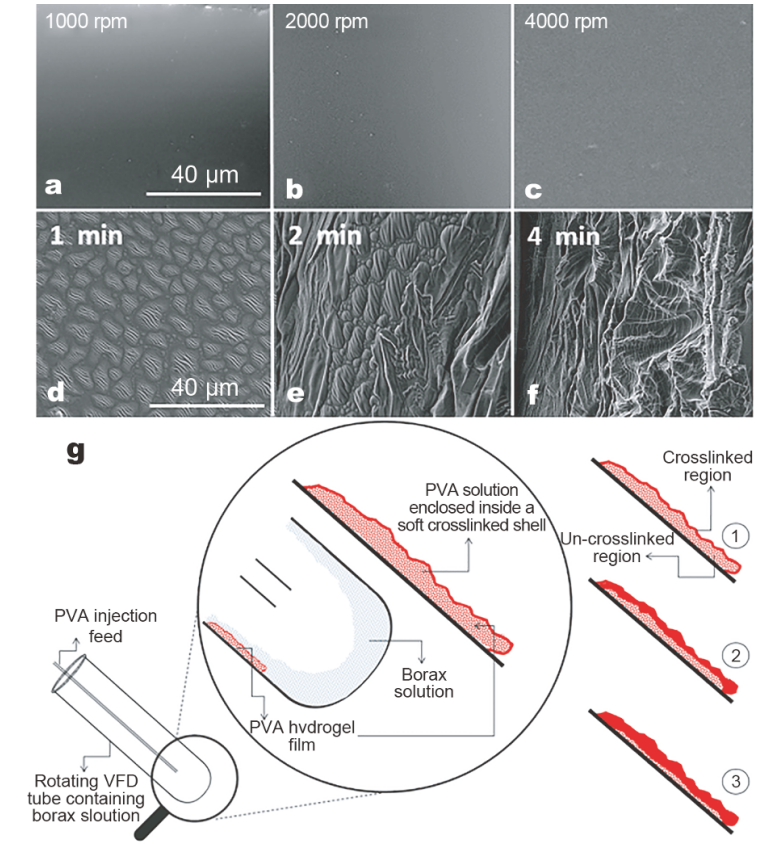

Figure $5(a-c)$ SEM images of the PVA hydrogel films captured from their back layers at different rotation speeds. $(\mathrm{d}-\mathrm{f})$ SEM images of the surface of the PVA hydrogel films that were crosslinked at the time points of 1,2 and $4 \mathrm{~min}$ at the rotation speed of $4000 \mathrm{rpm}$. (a-f) use the same scale bars. (g) A schematic illustration of the mechanism of PVA hydrogel formation using VFD. A magnified cross-section of the VFD tube after injection of PVA solution into the rotating borax solution reveals the formation of a core-shell structure, with the outer surface of the PVA solution first to contact the borax solution. The diffusion of borax over time (1)-(3) into the core-shell PVA film resulted in the formation of PVA hydrogel film.

gel, leading to the formation of hydrogel films with different morphologies. At the beginning of the crosslinking process, the crosslinked shell (outer surface) was soft and therefore the distribution of the centrifugal force resulted in the formation of a texture on the surface of the PVA hydrogel film. Because the centrifugal force was proportional to the rotating speed, any specific rotation speed has an impact, leading to the formation of unique surface morphology.

\section{Significantly enhanced self-healing properties of PVA hydrogels under VFD formation}

We found that a decrease in the concentration of the crosslinking agent, borax in this study, led to the production of a PVA-based solution with a strong selfhealing property. Our observation confirmed that at $4000 \mathrm{rpm}$ rotation of the VFD tube, the use of $50 \mathrm{mmol} \mathrm{L}^{-1}$ borax resulted in the formation of a highly extensible PVA hydrogel with improved self-adhesion property (Supplementary movies $2-5$ ). In contrast, tra- 
ditionally prepared PVA hydrogel (mixing of borax and PVA solution without the employment of VDF) displayed both strong self-adhesion and low extensibility. By quantifying the self-adhesion stress at the cutting edge of the gel, our experimental results revealed that detachment occurred at $40 \%$ strain for the traditionally prepared PVA hydrogel, which was significantly lower than that of the VFD-driven PVA hydrogel prepared at $4000 \mathrm{rpm}$. Detachment between the cutting edges of the VFD-driven PVA hydrogel was not seen even at $250 \%$ strain (Fig. 6). Interestingly, we found that after application of $5 \% \mathrm{~s}^{-1}$ strain for $260 \mathrm{~s}$, the VFD-driven PVA hydrogel experienced $5.6 \mathrm{mN} \mathrm{mm}^{-2}$ fracture stress while it was still attached. The fracture stress for the traditionally prepared PVA hydrogel was approximately $3.5 \mathrm{mN} \mathrm{mm}^{-2}$ at the breaking point, which occurred after the application of strain for $120 \mathrm{~min}$. A $60 \%$ improvement in fracture stress was seen when VFD was employed for the fabrication of PVA-based self-healing hydrogel, compared with the hydrogels that were prepared using the traditional approach. This observation can be explained based on the formation of a more homogeneous structure when the VFD was employed. The precise mixing of PVA and borax solution, which was readily achievable by using the VFD, led to better spatial distribution of borax molecules within the PVA chains. This, in turn, resulted in the formation of a more uniform structure that was not identical to that of PVA hydrogel prepared by the traditional method. In fact, the propagation of borax molecules within the PVA chains was not uniform when a traditional method was recruited, and it was more likely that this simple mixing led to the agglomeration of borax. While the conventional batch techniques, such as high shear mixing reaction are less likely to provide a platform of micro-mixing and shear force to simultaneously form a uniform distribution of components at the micro-scale, the result of the current study may motivate other investigations to address the similarities and differences between different techniques for fabrication of self-healing hydrogels. Therefore, at the micro-level, a more heterogeneous structure consisting of aggregated borax molecules was formed, which was responsible for the lower self-healing properties (Fig. 6h). The associated movies (Supplementary movies 6 and 7) indicate the selfhealing property of the PVA-based hydrogels during the measurement of fracture stress.

\section{CONCLUSIONS}

One-step preparation of PVA-based hydrogel films under thin film formation is reported in this study, providing a
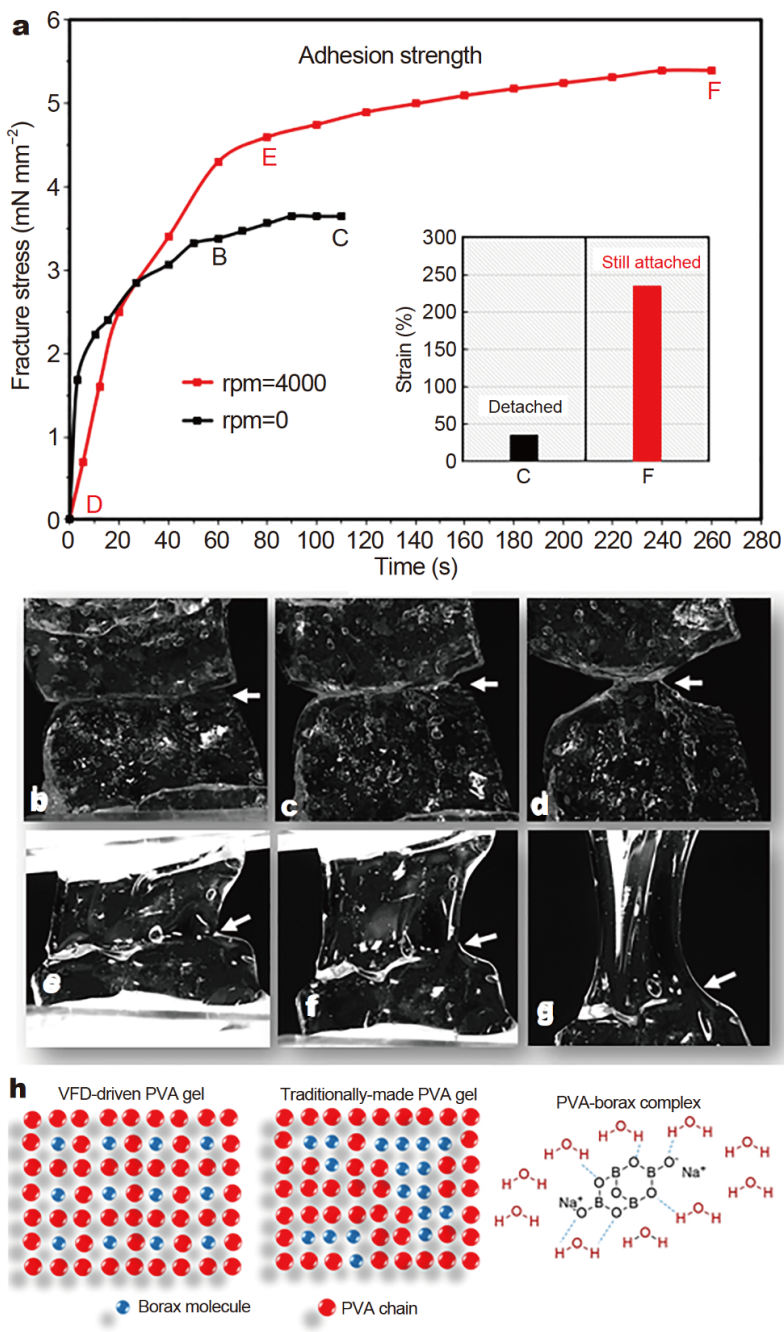

Figure 6 (a) Comparison of the fracture strength and extensibility of PVA-based hydrogels prepared traditionally and using a VDF. The magnified images $(\times 4)$ were captured during the measurement of the self-adhesion property of (b-d) traditionally prepared and (e-g) VFDdriven PVA-based hydrogels. White arrows identify the cutting edges during the test. To execute the experiment, the cutting edges were reattached before extension, as already explained. (h) Chemical structure and schematic drawing the PVA-borax complex, indicating the homogeneous structure of the VFD-driven PVA gel compared with the heterogeneous structure including aggregation of borax molecules in the traditionally made PVA hydrogel.

new strategy for controlling the surface morphologies during the preparation of hydrogels. By changing both rotation speed and crosslinking density during the preparation of hydrogels, the texture of the surface, as well as their self-healing property, could be significantly tuned, with higher speeds significantly increasing the extensibility and self-fracture stress. Direct control of surface morphology, as an advantage of the employment of 
VFD, opens new opportunities for biological and material studies.

\section{Received 28 January 2020; accepted 16 March 2020; published online 15 April 2020}

1 Cascone S, Lamberti G. Hydrogel-based commercial products for biomedical applications: A review. Int J Pharm, 2020, 573: 118803

2 Tavakoli J, Zhang H, Tang BZ, et al. Aggregation-induced emission lights up the swelling process: A new technique for swelling characterisation of hydrogels. Mater Chem Front, 2019, 3: 664-667

3 Tavakoli J, Gascooke J, Xie N, et al. Enlightening freeze-thaw process of physically cross-linked poly(vinyl alcohol) hydrogels by aggregation-induced emission fluorogens. ACS Appl Polym Mater, 2019, 1: 1390-1398

4 Tavakoli J, Tang Y. Hydrogel based sensors for biomedical applications: An updated review. Polymers, 2017, 9: 364

5 Jia R, Li L, Ai Y, et al. Self-healable wire-shaped supercapacitors with two twisted $\mathrm{NiCo}_{2} \mathrm{O}_{4}$ coated polyvinyl alcohol hydrogel fibers. Sci China Mater, 2018, 61: 254-262

6 Zhang X, Xia LY, Chen X, et al. Hydrogel-based phototherapy for fighting cancer and bacterial infection. Sci China Mater, 2017, 60: 487-503

7 Tavakoli J, Mirzaei S, Tang Y. Cost-effective double-layer hydrogel composites for wound dressing applications. Polymers, 2018, 10: 305

8 Yan W, Chen Q, Meng X, et al. Multicycle photocatalytic reduction of $\mathrm{Cr}(\mathrm{VI})$ over transparent $\mathrm{PVA} / \mathrm{TiO}_{2}$ nanocomposite films under visible light. Sci China Mater, 2017, 60: 449-460

9 Li SK, Mao LB, Gao HL, et al. Bio-inspired clay nanosheets/ polymer matrix/mineral nanofibers ternary composite films with optimal balance of strength and toughness. Sci China Mater, 2017, 60: 909-917

10 Zhong $\mathrm{L}$, Qu $\mathrm{Y}$, Shi $\mathrm{K}$, et al. Biomineralized polymer matrix composites for bone tissue repair: A review. Sci China Chem, 2018, 61: 1553-1567

11 Baker MI, Walsh SP, Schwartz Z, et al. A review of polyvinyl alcohol and its uses in cartilage and orthopedic applications. J Biomed Mater Res, 2012, 100: 1451-1457

12 Slaughter BV, Khurshid SS, Fisher OZ, et al. Hydrogels in regenerative medicine. Adv Mater, 2009, 21: 3307-3329

13 Costa-Júnior ES, Barbosa-Stancioli EF, Mansur AAP, et al. Preparation and characterization of chitosan/poly(vinyl alcohol) chemically crosslinked blends for biomedical applications. Carbohydr Polym, 2009, 76: 472-481

14 Dehbari N, Tavakoli J, Zhao J, et al. In situ formed internal water channels improving water swelling and mechanical properties of water swellable rubber composites. J Appl Polym Sci, 2017, 134: 44548

15 Liu J, Ye J, Pan F, et al. Solid-state yet flexible supercapacitors made by inkjet-printing hybrid ink of carbon quantum dots/graphene oxide platelets on paper. Sci China Mater, 2019, 62: 545-554

16 Chee BS, Goetten de Lima G, Devine DM, et al. Investigation of the effects of orientation on freeze/thawed polyvinyl alcohol hydrogel properties. Mater Today Commun, 2018, 17: 82-93

17 Peppas NA, Scott JE. Controlled release from poly(vinyl alcohol) gels prepared by freezing-thawing processes. J Control Release, 1992, 18: 95-100

18 Hassan CM, Peppas NA. Cellular PVA hydrogels produced by freeze/thawing. J Appl Polym Sci, 2000, 76: 2075-2079
19 Mansur HS, Sadahira CM, Souza AN, et al. FTIR spectroscopy characterization of poly(vinyl alcohol) hydrogel with different hydrolysis degree and chemically crosslinked with glutaraldehyde. Mater Sci Eng-C, 2008, 28: 539-548

20 ossipov da, hilborn j. Poly(vinyl alcohol)-based hydrogels formed by "click chemistry". Macromolecules, 2006, 39: 1709-1718

21 Schmedlen RH, Masters KS, West JL. Photocrosslinkable polyvinyl alcohol hydrogels that can be modified with cell adhesion peptides for use in tissue engineering. Biomaterials, 2002, 23: 4325-4332

22 Han J, Lei T, Wu Q. Facile preparation of mouldable polyvinyl alcohol-borax hydrogels reinforced by well-dispersed cellulose nanoparticles: Physical, viscoelastic and mechanical properties. Cellulose, 2013, 20: 2947-2958

23 Tavakoli J. Physico-mechanical, morphological and biomedical properties of a novel natural wound dressing material. J Mech Behav BioMed Mater, 2017, 65: 373-382

24 Tavakoli J, Tang Y. Honey/PVA hybrid wound dressings with controlled release of antibiotics: Structural, physico-mechanical and in-vitro biomedical studies. Mater Sci Eng-C, 2017, 77: 318325

25 Han J, Lei T, Wu Q. High-water-content mouldable polyvinyl alcohol-borax hydrogels reinforced by well-dispersed cellulose nanoparticles: Dynamic rheological properties and hydrogel formation mechanism. Carbohydr Polym, 2014, 102: 306-316

26 Spoljaric S, Salminen A, Luong ND, et al. Stable, self-healing hydrogels from nanofibrillated cellulose, poly(vinyl alcohol) and borax via reversible crosslinking. Eur Polym J, 2014, 56: 105-117

27 Cencetti C, Bellini D, Pavesio A, et al. Preparation and characterization of antimicrobial wound dressings based on silver, gellan, PVA and borax. Carbohydrate Polyms, 2012, 90: 1362-1370

28 Balakrishnan B, Jayakrishnan A. Self-cross-linking biopolymers as injectable in situ forming biodegradable scaffolds. Biomaterials, 2005, 26: 3941-3951

29 Yang N, Qi P, Ren J, et al. Polyvinyl alcohol/silk fibroin/borax hydrogel ionotronics: A highly stretchable, self-healable, and biocompatible sensing platform. ACS Appl Mater Interfaces, 2019, 11: $23632-23638$

30 Jian M, Wang C, Wang Q, et al. Advanced carbon materials for flexible and wearable sensors. Sci China Mater, 2017, 60: 10261062

31 Ochiai H, Fukushima S, Fujikawa M, et al. Mechanical and thermal properties of poly(vinyl alcohol) crosslinked by borax. Polym J, 1976, 8: 131-133

32 Lu B, Lin F, Jiang X, et al. One-pot assembly of microfibrillated cellulose reinforced PVA-borax hydrogels with self-healing and pH-responsive properties. ACS Sustain Chem Eng, 2017, 5: 948956

33 Lawrence MB, Joseph J, Phondekar K, et al. D.C. conductivity behaviour of poly(vinyl alcohol)-based ferrogels: role of borax and carbonyl iron. Polym Bull, 2019, 77: 6327-6341

34 Ge W, Cao S, Shen F, et al. Rapid self-healing, stretchable, moldable, antioxidant and antibacterial tannic acid-cellulose nanofibril composite hydrogels. Carbohydr Polym, 2019, 224: 115147

35 Jing Z, Xu A, Liang YQ, et al. Biodegradable poly(acrylic acid-coacrylamide)/poly(vinyl alcohol) double network hydrogels with tunable mechanics and high self-healing performance. Polymers, 2019, 11: 952

36 Lei W, Qi S, Rong Q, et al. Diffusion-freezing-induced microphase separation for constructing large-area multiscale structures on hydrogel surfaces. Adv Mater, 2019, 31: 1808217 
Britton J, Stubbs KA, Weiss GA, et al. Frontispiece: Vortex fluidic chemical transformations. Chem Eur J, 2017, 23: 13270-13278

38 Yasmin L, Chen X, Stubbs KA, et al. Optimising a vortex fluidic device for controlling chemical reactivity and selectivity. Sci Rep, 2013, 3: 2282

39 Vimalanathan K, Gascooke JR, Suarez-Martinez I, et al. Fluid dynamic lateral slicing of high tensile strength carbon nanotubes. Sci Rep, 2016, 6: 22865

40 Luo X, Al-Antaki AHM, Vimalanathan K, et al. Laser irradiated vortex fluidic mediated synthesis of luminescent carbon nanodots under continuous flow. React Chem Eng, 2018, 3: 164-170

41 Luo X, Smith P, Raston CL, et al. Vortex fluidic device-intensified aqueous two phase extraction of C-phycocyanin from Spirulina maxima. ACS Sustain Chem Eng, 2016, 4: 3905-3911

42 Luo X, Al-Antaki AHM, Pye S, et al. High-shear-imparted tunable fluorescence in polyethylenimines. ChemPhotoChem, 2018, 2: 343-348

43 Chen X, Dobson JF, Raston CL. Vortex fluidic exfoliation of graphite and boron nitride. Chem Commun, 2012, 48: 3703-3705

44 Yuan TZ, Ormonde CFG, Kudlacek ST, et al. Shear-stress-mediated refolding of proteins from aggregates and inclusion bodies. ChemBioChem, 2015, 16: 393-396

45 Tavakoli J, Pye S, Reza AHMM, et al. Tuning aggregation-induced emission nanoparticle properties under thin film formation. Mater Chem Front, 2020, 4: 537-545

46 Nune KC, Li S, Misra RDK. Advancements in three-dimensional titanium alloy mesh scaffolds fabricated by electron beam melting for biomedical devices: Mechanical and biological aspects. Sci China Mater, 2018, 61: 455-474

47 Lei D, Luo B, Guo Y, et al. 4-axis printing microfibrous tubular scaffold and tracheal cartilage application. Sci China Mater, 2019, 62: 1910-1920

48 Fan F, Sun J, Chen B, et al. Rotating magnetic field-controlled fabrication of magnetic hydrogel with spatially disk-like microstructures. Sci China Mater, 2018, 61: 1112-1122

49 Tavakoli J, Laisak E, Gao M, et al. Aiegen quantitatively monitoring the release of $\mathrm{Ca}^{2+}$ during swelling and degradation process in alginate hydrogels. Mater Sci Eng-C, 2019, 104: 109951

Acknowledgements Tavakoli J and Tang Y acknowledge an International Research Grant (International Laboratory for Health Technologies) of South Australia for support. Raston CL is grateful for support from the Australian Research Council and Ma Y is grateful for the support from the National Natural Science Foundation of China (51679183). The expertise, equipment, and support provided by Microscopy Australia and the Australian National Fabrication Facility at the South Australian nodes under the National Collaborative Research Infrastructure Strategy are acknowledged.

Author contributions The study was designed by Tavakoli J and Tang Y. Experiment and data collection were performed by Tavakoli J and Ma Y. All authors contributed to the data analysis and general discussion. The manuscript was written by Tavakoli J and was critically reviewed by
Tang Y and Raston CL.

Conflict of interest The authors declare that they have no conflict of interest.

Supplementary information Supporting movies are available in the online version of the paper.

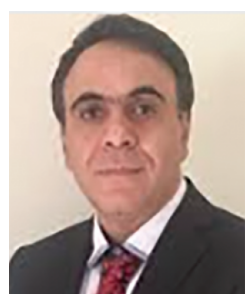

Javad Tavakoli obtained his $\mathrm{PhD}$ degree in Flinders University, Australia in 2018. Currently, he is a Chancellor's postdoctoral Research Fellow at the University of Technology Sydney, Australia. His main research interests are multiscale characterization and fabrication of smart materials including hydrogels for biomedical applications.

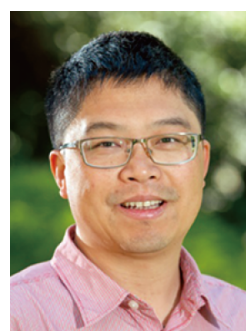

Youhong Tang obtained his $\mathrm{PhD}$ degree from the Hong Kong University of Science and Technology in 2007. He moved to Flinders University with an ARC-DECRA in 2012 from the Centre for Advanced Materials Technology, the University of Sydney. As a material science and engineering researcher, his research interests mainly focus on the structure-process-property relations of polymeric materials and nanocomposites, multifunctional and value-added nanocomposites and bioresources, biomaterials and biosensors, especially incorporating novel aggregation-induced emission materials.

\section{泞流介导的一步法制备具有可调表面形态和增强 自愈性能的聚乙烯醇水凝胶膜}

Javad Tavakoli ${ }^{1,2^{*}}$, Colin L. Raston ${ }^{2}$, 马勇 ${ }^{3,2}$, 唐友宏 ${ }^{2^{*}}$

摘要 控制基于聚乙烯醇(PVA)的水凝胶表面形态的传统策略包 括冷冻干燥和静电纺丝, 但是这些方法要求进行后处理过程, 这可 能会影响最终的水凝胶的质地和性能. 特别是, 使用这些方法几乎 不可能在PVA水凝胶的形成过程中控制其表面形貌. 本研究首次 报道了借助新颖的浴流装置(VFD)技术一步法制备PVA水凝胶膜. 使用VFD可以很容易地制造出具有不同表面形貌的PVA水凝胶. 并通过降低交联剂的浓度, 获得了具有增强断裂应力(比传统制造 的水凝胶大 $60 \%$ )的自愈水凝胶. 有趣的是, 在 $5 \% \mathrm{~s}^{-1}$ 的应变率下进 行的 $260 \mathrm{~s}$ 机械测试中, 相关的自愈特性保持不变. VFD可以有效地 调控基于PVA的水凝胶的表面形貌及其相关特性，尤其是水凝胶 的自愈特性. 